\title{
A DISCRETE PREDATOR-PREY SYSTEM WITH AGE-STRUCTURE FOR PREDATOR AND NATURAL BARRIERS FOR PREY*
}

\author{
SANYI TANG ${ }^{1}$ AND LANSUn Chen ${ }^{1}$
}

\begin{abstract}
We analyze a two species discrete predator-prey model in which the prey disperses between two patches of a heterogeneous environment with barriers and the mature predator disperses between the patches with no barrier. By using the discrete dynamical system generated by a monotone, concave maps for subcommunity of prey, we obtain the subcommunity of prey exists an equilibrium which attracts all positive solutions, and using the stability trichotomy results on the monotone and continuous operator, we obtain some sufficient conditions for the permanence of species. These results are applied to the models with rational growth functions and exponential growth functions. We also present numerical examples to illustrate the dynamic complexity of systems.
\end{abstract}

Mathematics Subject Classification. 34C35, 39A10.

Received: November 14, 2000. Revised: February 7, 2001.

\section{INTRODUCTION}

A population in which generations do not overlap or population size is relatively small is, in general, modeled by a discrete growth process. Nonlinear difference equations are often utilized to describe the dynamics of such populations where growth occurs in isolated steps $[2,9,18,24]$. The predator-prey system is an important population model and has been studied by many authors $[2,7,9,13,18,23,24]$. A standard example of discrete predator-prey system is the modified Nickoson-Bailey recurrence [2]

$$
\left\{\begin{aligned}
x(t+1) & =x(t) \exp \left(r-\frac{r}{k} x(t)-a y(t)\right) \\
y(t+1) & =b x(t)(1-\exp (-a y(t)))
\end{aligned}\right.
$$

where $a, b, r, k$ are positive constants. System (1.1) has a unique stationary point $(\bar{x}, \bar{y})$ with $\bar{x}>0, \bar{y}>0$ and system (1.1) exhibits different features(such as: stable focus, invariant curve, strange attractor) according to different values of the parameters, as described in $[2,9,18]$.

It is assumed in the classical predator-prey model that each individual predator admits the same ability to attack prey. However, in the natural world, this is not how things stand. In this paper, we classify individuals of predator as belonging to either the immature or the mature and suppose that the immature population does

\footnotetext{
Keywords and phrases. Age-structure, natural barrier, subcommunity, persistence.

* Supported by the National Natural Science Foundation of China

${ }^{1}$ Institute of Mathematics, Academy of Mathematics and System Sciences, Chinese Academy of Sciences, Beijing 100080,

P. R. China. e-mail: tsy@math08.math.ac.cn
} 
not feed on prey. This seems reasonable for a number of vertebrate and invertebrate. One of the purpose of this paper is to study how the age-structure influences on the dynamical behavior of solutions. Thus we consider:

$$
\left\{\begin{aligned}
x(t+1) & =x(t) g\left(x(t)+\beta y_{2}(t)\right) \\
y_{1}(t+1) & =x(t)\left[1-\frac{g\left(\beta y_{2}(t)\right)}{g(0)}\right] \\
y_{2}(t+1) & =b y_{1}(t)+s y_{2}(t)
\end{aligned}\right.
$$

where $x(t)$ is the prey population density at generation $t, y_{1}(t)$ and $y_{2}(t)$ are the densities of the immature and mature predator at generation $t$, respectively. $\beta$ is nonnegative constant, $0<b \leq 1$ is the fraction of individuals in the immature class that survives to mature, and $0 \leq s<1$ denotes the fraction of mature individuals that are alive in the mature class after one generation.

In addition, since the pioneering theoretical work by Skellam [20], Levin [15] and Okubo [17], many papers have focused on the effect of spatial factors which plays a crucial role in permanence and stability of population $[10,14,22]$. In fact, dispersal between patches often occurs in ecological environments, and more realistic models should include the dispersal process. The analysis of these models has been centered around the coexistence of populations and local (or global) stability of equilibria. There are many results on this respect in continuous system [7,13,22]. For discrete system, once diffusion is introduced, system becomes difficult to deal with, and asymptotic behavior of solution becomes very complex. For example, Hasting [10] discussed the following systems

$$
\left\{\begin{array}{l}
x_{1}(t+1)=\overline{x_{1}}(t)+d\left[\overline{x_{2}}(t)-\overline{x_{1}}(t)\right] \\
x_{2}(t+1)=\overline{x_{2}}(t)+d\left[\overline{x_{1}}(t)-\overline{x_{2}}(t)\right]
\end{array}\right.
$$

where $\bar{x}_{i}=f\left(r_{i}, x_{i}(t)\right)$ and $f\left(r_{i}, x_{i}(t)\right)=r_{i} x_{i}\left(1-x_{i}\right)$ or $x_{i} \exp \left(r_{i}\left(1-x_{i}\right)\right)$ for $i=1,2, d$ is the fraction of the population that is exchanged with $0 \leq d \leq 1 / 2$. By using both numerical and analytical approaches, Hasting demonstrated several surprising features, for example, passive dispersal can cause chaotic dynamics to be replaced by simple periodic dynamics (for some details of this, see [10]) .

In this paper, we consider the discrete predator-prey model with diffusion where there are barriers for dispersal among preys but not among predators. This is in accordance with the fact given in [14]. The prey are the numbers of the porcupine caribou herd that dwell in the northern Yukon Territory of Canada. Their dispersion carries them across rivers and mountains. These are clearly barriers to their dispersion. One of the predator population for this herd is the golden eagle. For these birds, the rivers and mountains do not form barriers. Another purpose of this paper is to investigate the effect of diffusion on asymptotic behavior of system. Let us consider:

$$
\left\{\begin{aligned}
x_{1}(t+1)= & x_{1}(t) g_{1}\left(x_{1}(t)+\beta_{1} x_{4}(t)\right)+d\left[x_{2}(t) g_{2}\left(x_{2}(t)+\beta_{2} x_{4}(t)\right)\right. \\
& \left.-x_{1}(t) g_{1}\left(x_{1}(t)+\beta_{1} x_{4}(t)\right)\right] \\
x_{2}(t+1)= & x_{2}(t) g_{2}\left(x_{2}(t)+\beta_{2} x_{4}(t)\right)+d\left[x_{1}(t) g_{1}\left(x_{1}(t)+\beta_{1} x_{4}(t)\right)\right. \\
& \left.-x_{2}(t) g_{2}\left(x_{2}(t)+\beta_{2} x_{4}(t)\right)\right] \\
x_{3}(t+1)= & x_{1}(t)\left[1-\frac{g_{1}\left(\beta_{1} x_{4}(t)\right)}{g_{1}(0)}\right]+x_{2}(t)\left[1-\frac{g_{2}\left(\beta_{2} x_{4}(t)\right)}{g_{2}(0)}\right] \\
x_{4}(t+1)= & b x_{3}(t)+s x_{4}(t)
\end{aligned}\right.
$$

where the diffusion coefficient $d$ is not more than $\frac{1}{2}, \beta_{i}(i=1,2)$ are nonnegative constants, for each $i \in\{1,2\}$, $x_{i}(t)$ is the prey population density at generation $t$, with the function $g_{i}$ (or $g$ in system $\left.(1.2)\right):[0,+\infty) \rightarrow$ $(0,+\infty)$ being its growth function. Also, $x_{3}(t)$ and $x_{4}(t)$ are the densities of the immature and mature predators at generation $t$, respectively. 
Pioneer species are species that thrive at very small population sizes when left to their own natural devices with no interference [19]. Thus, if prey $i(i=1,2)$ is a pioneer species, then $g_{i}(0)($ or $g(0))>1$. To make each prey $i$ in system (1.4) be a pioneer species and to achieve self-regulation of the prey species, it is assumed throughout the present study that the growth functions $g_{i}$ (or $\left.g\right)$ are strictly decreasing smooth functions with respect to $\left(x_{i}+\beta_{i} x_{4}\right)(i=1,2)$.

Frank et al. [24] has considered this kinds of systems, they mainly investigated the persistence of dominant species and extinction of species with a safe refuge. However, in this paper, we consider the strong(weak) persistence and permanence of all species and obtain that diffusion has no effect on persistence and permanence of systems. Meanwhile, we show that asymptotic behavior of solutions of system (1.2) in permanent region is much more complex than that in corresponding system without age-structure. By numerical simulation, we show that except that some features such as stable focus, period-double bifurcation which are found in corresponding system without age-structure, some new and interesting features, for example, chaos suddenly appears from steady state as parameter varies, are found. This suggests that age-structure can make the behavior of discrete system more complex.

The organization of this paper is as follows. In the next section, we present conditions for the boundedness of system (1.4). In Section 3, we consider the subcommunity of prey of systems (1.2) and (1.4). In Section 4, some definitions on persistence are given and the strong persistence and weak persistence of populations are investigated. In Section 5, sufficient conditions for permanence of systems (1.2) and (1.4) are obtained by exploiting the main theorem in $[4,8]$ and the stability trichotomy results on monotone and continuous operator in [12]. Section 6 gives some applications of the main theorems and numerical results which show the dynamic behavior is very complex in the permanent region. In the final discussion section, we try to interpret our mathematical results in terms of their ecological implications and formulate conclusions. We also point out some future research directions.

\section{BOUNDEDNESS}

In system (1.4), for each $i \in\{1,2\}$, we define the map $h_{i}:[0,+\infty) \rightarrow(0,+\infty)$ by $h_{i}\left(x_{i}\right)=x_{i} g_{i}\left(x_{i}\right)$. The iterates of the one-dimensional map $h_{i}$ are the density sequence generated by the single prey species ecological model $x_{i}(t+1)=x_{i}(t) g_{i}\left(x_{i}(t)\right)(i=1,2$,$) . We denote the positive fixed point of h_{i}$ by $x_{i}^{*}(i=1,2)$. Since each growth function $g_{i}$ takes on the value one in $(0,+\infty), x_{i}^{*}$ exists in $(0,+\infty)$.

For each $i \in\{1,2\}$, if $0<x_{i}<x_{i}^{*}$, then $h_{i}\left(x_{i}\right)>x_{i}$; and if $x_{i}>x_{i}^{*}$, then $h_{i}\left(x_{i}\right)<x_{i}$. Consequently, under $h_{i}$ iterations, one see that $I_{i}=h_{i}\left(\left[0, x_{i}^{*}\right]\right)$ is a compact invariant interval in $R_{+}$into which every point either eventually enters and stays or just limits on it .

To write system (1.4) as a map, we define the map $F: R_{+}^{4} \rightarrow R_{+}^{4}$

$$
\left\{\begin{array}{l}
F_{1}(x)=x_{1} g_{1}\left(x_{1}+\beta_{1} x_{4}\right)+d\left[x_{2} g_{2}\left(x_{2}+\beta_{2} x_{4}\right)-x_{1} g_{1}\left(x_{1}+\beta_{1} x_{4}\right)\right] \\
F_{2}(x)=x_{2} g_{2}\left(x_{1}+\beta_{2} x_{4}\right)+d\left[x_{1} g_{1}\left(x_{1}+\beta_{1} x_{4}\right)-x_{2} g_{2}\left(x_{2}+\beta_{2} x_{4}\right)\right] \\
F_{3}(x)=x_{1}\left[1-\frac{g_{1}\left(\beta_{1} x_{4}\right)}{g_{1}(0)}\right]+x_{2}\left[1-\frac{g_{2}\left(\beta_{2} x_{4}\right)}{g_{2}(0)}\right] \\
F_{4}(x)=b x_{3}+s x_{4} .
\end{array}\right.
$$

The set of all iterates of the map $F$ is equivalent to the set of all density sequences generated by system (1.4). $F^{t}(x)$ is the map evaluated at the point $x=\left(x_{1}, x_{2}, x_{3}, x_{4}\right) \in R_{+}^{4}$. Consequently, in system (1.4), $F^{t}$ describes the population densities in generation $t$.

We will use the following auxiliary result to establish the boundedness of system (1.4). 
Lemma 2.1. [1]. Let $Y(t)=\left(Y_{1}(t), \cdots, Y_{m}(t)\right)^{T}, F(Y(t))=\left(F_{1}(Y(t)), \cdots, F_{m}(Y(t))\right)^{T}$ and $Y(t+1)=$ $F(Y(t))$. If the following three conditions hold:

(i) $X\left(t_{1}\right) \geq Y\left(t_{1}\right)$,

(ii) $F(X) \geq F(Y)$ if $X \geq Y$ and

(iii) $X(t+1) \geq F(X(t)), t=t_{1}, t_{1}+1, \cdots$

Then $X(t) \geq Y(t), t=t_{1}, t_{1}+1, \cdots$.

Proof. The proof follows by induction on $t$. A similar result holds if all of the inequalities are reversed.

Theorem 2.1. In system (1.4), every point has a bounded orbit.

Proof. For each $i \in\{1,2,3,4\}$ and each point $x \in R_{+}^{4}$, we need to show that each of the sequence $\left\{F_{i}^{t}(x)\right\}_{t \geq 0}$ is bounded.

First, we show that

$$
\max \left\{F_{1}(x), F_{2}(x)\right\} \leq \max \left\{h_{1}\left(x_{1}\right), h_{2}\left(x_{2}\right)\right\} .
$$

From (2.1), one see that

$$
F_{1}(x)=(1-d) x_{1} g_{1}\left(x_{1}+\beta_{1} x_{4}\right)+d x_{2} g_{2}\left(x_{2}+\beta_{2} x_{4}\right)
$$

and

$$
F_{2}(x)=d x_{1} g_{1}\left(x_{1}+\beta_{1} x_{4}\right)+(1-d) x_{2} g_{2}\left(x_{2}+\beta_{2} x_{4}\right) .
$$

Therefore, if $x_{1} g_{1}\left(x_{1}+\beta_{1} x_{4}\right) \geq x_{2} g_{2}\left(x_{2}+\beta_{2} x_{4}\right)$, then

$$
\max \left\{F_{1}(x), F_{2}(x)\right\} \leq x_{1} g_{1}\left(x_{1}+\beta_{1} x_{4}\right) .
$$

On the other hand, if $x_{1} g_{1}\left(x_{1}+\beta_{1} x_{4}\right) \leq x_{2} g_{2}\left(x_{2}+\beta_{2} x_{4}\right)$, then

$$
\max \left\{F_{1}(x), F_{2}(x)\right\} \leq x_{2} g_{2}\left(x_{2}+\beta_{2} x_{4}\right) .
$$

By the monotonicity conditions on the growth functions $g_{i}(i=1,2)$, we have

$$
x_{i} g_{i}\left(x_{i}+\beta_{i} x_{4}\right) \leq x_{i} g_{i}\left(x_{i}\right) \quad \text { for } \quad i=1,2 .
$$

Consequently

$$
\max \left\{F_{1}(x), F_{2}(x)\right\} \leq \max \left\{h_{1}\left(x_{1}\right), h_{2}\left(x_{2}\right)\right\} .
$$

Recall that $I_{i} \equiv h_{i}\left(\left[0, x_{i}^{*}\right]\right)(i=1,2)$ are invariant sets under $h_{i}$ iterates, respectively. Moreover, if $x_{i}>$ $\max I_{i}$, then $h_{i}\left(x_{i}\right)<x_{i}$. Hence, the sequence $\left\{F_{1}^{t}(x)\right\}_{t \geq 0}$ and $\left\{F_{2}^{t}(x)\right\}_{t \geq 0}$ are bounded. In addition, $F_{3}(x)=$ $\sum_{i=1}^{2} x_{i}\left[1-\frac{g_{i}\left(\beta_{i} x_{4}\right)}{g_{i}(0)}\right] \leq \sum_{i=1}^{2} x_{i}$. Hence, the sequence $\left\{F_{3}^{t}(x)\right\}_{t \geq 0}$ is also bounded. That is, there exist $t_{1}>0$ and constant $B>0$ such that $\max \left\{x_{1}(t), x_{2}(t)\right\} \leq B$ and $y_{1}(t) \leq 2 B$ for $t \geq t_{1}$. By induction and Lemma 2.1, $y_{2}(t)$ is bounded above by the solution $z(t)$ of the one-dimensional map

$$
z(t+1)=B+s z(t), \quad z\left(t_{1}\right)=y_{2}\left(t_{1}\right), \quad t \geq t_{1} .
$$


The solution of this map is given by

$$
z(t)=\frac{s^{t-t_{1}}[(1-s) z(t)-B]+B}{1-s} .
$$

Since $0 \leq s<1, z(t)$ is monotonically decreasing to $\frac{B}{1-s}$ if $z\left(t_{1}\right) \geq \frac{B}{1-s}$ and monotonically increasing to $\frac{B}{1-s}$ if $z\left(t_{1}\right)<\frac{B}{1-s}$. Therefore all orbits of model (1.4) will eventually enter and remain in the rectangle

$$
[0, B] \times[0, B] \times[0,2 B] \times\left[0, \frac{B}{1-s}\right]
$$

Corollary 2.1. Every point in system (1.2) has a bounded orbit.

\section{Subcommunity of Prey}

First, we consider the single-species dynamics of the prey of system (1.2) in the absence of predator. This dynamics is given by the equation

$$
x(t+1)=h\left(x_{t}\right)=x(t) g(x(t))
$$

The dynamics of system (3.1) is discussed in [6]. Let $\hat{x}=\max I$ where $I=h\left(\left[0, x^{*}\right]\right)$ and $x^{*}$ is the fixed point of map $h$. Then $\hat{x} \geq x^{*}$ and if $\{x(t)\}_{t \in Z_{+}}$is a positive orbit of system (3.1), then $\limsup _{t \rightarrow \infty} x(t) \leq \hat{x}$. Further, by the properties of $g(x)$, it is shown in [6] that there exists $\eta>0, \eta$ computable, such that for $x(0)>0, M=\{x \mid \eta \leq x \leq \hat{x}\}$ is the maximal invariant set of system (3.1).

Now we can define that $n_{1}=-\frac{b \beta \eta g^{\prime}(0)}{(1-s) g(0)}$ is the intrinsic net reproductive number (for some details of this, see [5]) of system (1.2). Since $-\frac{\beta g^{\prime}(0)}{g(0)}$ is the maximal searching efficiency of prey, then $n_{1}$ is the expected value of offspring per adult individual per lifetime, which plays an important role in the persistence properties of system (1.2).

For system (1.4), we fix $x_{4}=0$ and consider the subcommunity consisting of prey $x_{1}$ and $x_{2}$ :

$$
\left\{\begin{array}{l}
x_{1}(t+1)=x_{1}(t) g_{1}\left(x_{1}(t)\right)+d\left[x_{2}(t) g_{2}\left(x_{2}(t)\right)-x_{1}(t) g_{1}\left(x_{1}(t)\right)\right] \\
x_{2}(t+1)=x_{2}(t) g_{2}\left(x_{2}(t)\right)+d\left[x_{1}(t) g_{1}\left(x_{1}(t)\right)-x_{2}(t) g_{2}\left(x_{2}(t)\right)\right] .
\end{array}\right.
$$

From (2.1), we have

$$
\left\{\begin{array}{l}
F_{1}\left(x_{1}, x_{2}\right)=x_{1} g_{1}\left(x_{1}\right)+d\left[x_{2} g_{2}\left(x_{2}\right)-x_{1} g_{1}\left(x_{1}\right)\right]=(1-d) h_{1}\left(x_{1}\right)+d h_{2}\left(x_{2}\right) \\
F_{2}\left(x_{1}, x_{2}\right)=x_{2} g_{2}\left(x_{2}\right)+d\left[x_{1} g_{1}\left(x_{1}\right)-x_{2} g_{2}\left(x_{2}\right)\right]=d h_{1}\left(x_{1}\right)+(1-d) h_{2}\left(x_{2}\right) .
\end{array}\right.
$$

Let

$S=\left\{f(x) \in C^{2}[0, \infty) \rightarrow[0, \infty): f^{\prime}(x)>0, f^{\prime \prime}(x)<0, f^{\prime}(0)>1, f(0)=0\right.$, and $f(x)=x$ for some $\left.x>0\right\}$

Before stating our main theorem in this section, we need the following Lemma:

Lemma 3.1. [21]. Let $T: R_{+}^{n} \rightarrow R_{+}^{n}$ be continuous, $C^{1}$ in $\operatorname{int}\left(R_{+}^{n}\right)$, and suppose DT(0) exists with $\lim _{x \rightarrow 0, x>0} D T(x)=D T(0)$, in addition, assume

(a) $D T(x)>0$ if $x>0$

(b) $D T(y) \leq D T(x)(\neq D T(x))$ if $0<x<y$. 
If $T(0)=0$, let $\lambda=\rho(D T(0))$, if $\lambda \leq 1$ then for every $x \geq 0, T^{n} x \rightarrow 0$ as $n \rightarrow \infty$; If $\lambda>1$ then either $T^{n} x \rightarrow \infty$ as $n \rightarrow \infty$ for every $x>0$ or there exists a unique nonzero fixed point $q$ of $T$. In the later case, $q>0$ and for every $x \geq 0(\neq 0), T^{n} x \rightarrow q$ as $n \rightarrow \infty$.

If $T(0) \neq 0$, then either $T^{n} x \rightarrow 0$ as $n \rightarrow \infty$ for every $x \geq 0$ or there exists a unique nonzero fixed point $q$ of $T$. In the later case, $q>0$ and for every $x \geq 0(\neq 0), T^{n} x \rightarrow q$ as $n \rightarrow \infty$.

Theorem 3.1. If $h_{i}\left(x_{i}\right) \in S(i=1,2)$. Then there exists a unique positive fixed point $q=\left(q_{1}, q_{2}\right)$ of system (3.2) and for every $x=\left(x_{1}, x_{2}\right)>0$

$$
F_{1}^{n}\left(x_{1}, x_{2}\right) \rightarrow q_{1}, \quad F_{2}^{n}\left(x_{1}, x_{2}\right) \rightarrow q_{2}, \quad \text { for } \quad n \rightarrow \infty .
$$

Proof. First, we show that $F\left(x_{1}, x_{2}\right)$ satisfies all the hypotheses of Lemma 3.1. For every $x=\left(x_{1}, x_{2}\right)>0$, from $(3.3), F_{1}(x)=(1-d) h_{1}\left(x_{1}\right)+d h_{2}\left(x_{2}\right)$ and $F_{2}(x)=d h_{1}\left(x_{1}\right)+(1-d) h_{2}\left(x_{2}\right)$. Since $h_{i}\left(x_{i}\right) \in S(i=1,2)$, then $F\left(x_{1}, x_{2}\right): R_{+}^{2} \rightarrow R_{+}^{2}$ be continuous, $C^{1}$ in $\operatorname{int}\left(R_{+}^{2}\right)$, and $F(0,0)=0$. Since

$$
\begin{aligned}
D F\left(x_{1}, x_{2}\right) & =\left(\begin{array}{ll}
\frac{\partial F_{1}}{\partial x_{1}} & \frac{\partial F_{1}}{\partial x_{2}} \\
\frac{\partial F_{2}}{\partial x_{1}} & \frac{\partial F_{2}}{\partial x_{2}}
\end{array}\right)=\left(\begin{array}{cc}
(1-d) h_{1}^{\prime}\left(x_{1}\right) & d h_{2}^{\prime}\left(x_{2}\right) \\
d h_{1}^{\prime}\left(x_{1}\right) & (1-d) h_{2}^{\prime}\left(x_{2}\right)
\end{array}\right) \\
& =\left(\begin{array}{cc}
(1-d)\left[g_{1}\left(x_{1}\right)+x_{1} g_{1}^{\prime}\left(x_{1}\right)\right] & d\left[g_{2}\left(x_{2}\right)+x_{2} g_{2}^{\prime}\left(x_{2}\right)\right] \\
d\left[g_{1}\left(x_{1}\right)+x_{1} g_{1}^{\prime}\left(x_{1}\right)\right] & (1-d)\left[g_{2}\left(x_{2}\right)+x_{2} g_{2}^{\prime}\left(x_{2}\right)\right]
\end{array}\right) .
\end{aligned}
$$

It follows that

$$
D F(0,0)=\left(\begin{array}{cc}
(1-d) g_{1}(0) & d g_{2}(0) \\
d g_{1}(0) & (1-d) g_{2}(0)
\end{array}\right)
$$

exists with $\lim _{x \rightarrow 0, x>0} D F\left(x_{1}, x_{2}\right)=D F(0,0)$, and it is easy to know that:

(a) $D F\left(x_{1}, x_{2}\right)>0 \quad$ if $\left(x_{1}, x_{2}\right)>0$

(b) $D F\left(y_{1}, y_{2}\right) \leq D F\left(x_{1}, x_{2}\right)\left(\neq D F\left(x_{1}, x_{2}\right)\right) \quad$ if $0<\left(x_{1}, x_{2}\right)<\left(y_{1}, y_{2}\right)$.

Let $\lambda=\rho(D F(0,0))$, then we have

$$
\begin{aligned}
\lambda & =\frac{(1-d)\left[g_{1}(0)+g_{2}(0)\right]+\sqrt{(1-d)^{2}\left[g_{1}(0)+g_{2}(0)\right]^{2}-4(1-2 d) g_{1}(0) g_{2}(0)}}{2} \\
& =\frac{(1-d)\left[g_{1}(0)+g_{2}(0)\right]+\sqrt{(1-2 d)\left[g_{1}(0)-g_{2}(0)\right]^{2}+d^{2}\left[g_{1}(0)+g_{2}(0)\right]^{2}}}{2} \\
& \geq \frac{(1-d)\left[g_{1}(0)+g_{2}(0)\right]+d\left[g_{1}(0)+g_{2}(0)\right]}{2} \\
& =\frac{1}{2}\left[g_{1}(0)+g_{2}(0)\right]>1 .
\end{aligned}
$$

By Theorem 2.1 and Lemma 3.1, we can see that there is a unique nonzero fixed point $q=\left(q_{1}, q_{2}\right)$ of $F\left(x_{1}, x_{2}\right)$ such that

$$
\lim _{n \rightarrow \infty} F^{n}\left(x_{1}, x_{2}\right)=\left(q_{1}, q_{2}\right) \text { for all } \quad x \in \operatorname{int}\left(R_{+}^{2}\right) .
$$


Further, we can define that $n_{2}=-\frac{b}{1-s} \sum_{i=1}^{2} \frac{\beta_{i} q_{i} g_{i}^{\prime}(0)}{g_{i}(0)}$ is the intrinsic net reproductive number of system (1.4), which represents the similar meaning to $n_{1}$ and also plays an important role in the persistence properties of system (1.4).

Example 3.1. To illustrate our main results in this section, we consider the model with rational growth:

$$
\left\{\begin{array}{l}
x_{1}(t+1)=\frac{b_{1} x_{1}(t)}{1+x_{1}(t)}+d\left[\frac{b_{2} x_{2}(t)}{1+x_{2}(t)}-\frac{b_{1} x_{1}(t)}{1+x_{1}(t)}\right] \\
x_{2}(t+1)=\frac{b_{2} x_{2}(t)}{1+x_{2}(t)}+d\left[\frac{b_{1} x_{1}(t)}{1+x_{1}(t)}-\frac{b_{2} x_{2}(t)}{1+x_{2}(t)}\right]
\end{array}\right.
$$

where $h_{i}\left(x_{i}\right)=\frac{b_{i} x_{i}(t)}{1+x_{i}(t)}(i=1,2), g_{i}\left(x_{i}\right)=\frac{b_{i}}{1+x_{i}(t)}(i=1,2), b_{i}>1, d$ are positive constants for $i=1,2$, and $d \in[0,1 / 2]$. Then we have $g_{i}(0)=b_{i}>1$, and $x_{i}^{*}=b_{i}-1>0$ satisfies $h_{i}\left(x_{i}^{*}\right)=x_{i}^{*}$. It is easy to see that $h_{i}\left(x_{i}\right) \in S$. By Theorem 3.1, there exists a unique positive equilibrium $q=\left(q_{1}, q_{2}\right)$ of system (3.4) which attracts all positive solutions.

Remark 3.1. For single-species discrete model (1.3) with exponential or logistic growth functions diffusion can increase the stability of system, i.e., passive dispersal can cause chaotic dynamics to be replaced by simple periodic dynamics [10]. However for model (3.4) with rational growth function, we obtain that there is a unique positive steady state which is global attractiviting under appropriate conditions, which is in line with the properties of the correspond to single-species continuous model with diffusion.

\section{Strong Persistence AND Weak PeRsistence}

We begin by presenting some definitions that will be used in the rest of paper:

Definition 4.1. $[17,20]$.

(a) System (1.4) is strongly persistent at time $N$ if $x_{i}(t)>0, i=1,2,3,4$ for each $t=0,1, \cdots, N$;

(b) System (1.4) is weakly persistent at time $N$ if $x_{i}(t)>0, i=1,2,3,4$ for each $t=0,1, \cdots, N-1$, and $x_{1}(N)>0, x_{2}(N)>0, x_{3}(N) \geq 0, x_{4}(N) \geq 0$ and $x_{3}(N)+x_{4}(N)>0$

(c) System (1.4) is strongly persistent if it is strongly persistent at time $N$ for each $N=0,1, \cdots$, and $\lim _{t \rightarrow \infty} \sup x_{i}(t)>0, i=1,2,3,4$

(d) System (1.4) is weakly persistent if it is persistent,strongly or weakly, at time $N$ for each $N=0,1, \cdots$, and $\lim _{t \rightarrow \infty} \sup x_{i}(t)>0, i=1,2$ and $\lim _{t \rightarrow \infty} \sup \left(x_{3}(t)+x_{4}(t)\right)>0$

(e) System (1.4) is uniformly persistent if it is strongly persistent at time $N$ for each $N=0,1, \cdots$, and there exists a positive constant $\eta$ such that $\lim _{t \rightarrow \infty} \inf x_{i}(t)>\eta, i=1,2,3,4$;

(f) System (1.4) is permanent if it is uniform persistent and point dissipative.

Obviously, strong persistence implies weak persistence, uniform persistence implies strong persistence.

Theorem 4.1. Assume system (1.4) is strongly persistent at time 0, then system (1.4) is strongly persistent provided the intrinsic net reproductive number $n_{2}>1$.

Proof. Since system (1.4) is strongly persistent at time 0, by induction on $t$, it is easy to see that system (1.4) is strongly persistent at time $\mathrm{N}$ for each $N=1,2, \cdots$.

The characteristic polynomial at $(0,0,0,0)$ is

$$
P(\lambda)=\left(\lambda^{2}-\lambda s\right)\left[\lambda^{2}-(1-d)\left(g_{1}(0)+g_{2}(0)\right) \lambda+(1-2 d) g_{1}(0) g_{2}(0)\right] .
$$

Obviously $(-1)^{3} P(-1)=-(1+s)\left[1+(1-d)\left(g_{1}(0)+g_{2}(0)\right)+(1-2 d) g_{1}(0) g_{2}(0)\right]<0$ because of $0 \leq s<1$ and $g_{i}(0)>1, i=1,2$. By Jury criterion, it follows that $(0,0,0,0)$ is unstable. 
The characteristic polynomial at $\left(q_{1}, q_{2}, 0,0\right)$ is

$$
\begin{aligned}
P(\lambda)=\left[\lambda^{2}-\lambda s+b \sum_{i=1}^{2} \frac{\beta_{i} q_{i} g_{i}^{\prime}(0)}{g_{i}(0)}\right]\left\{\lambda^{2}-(1-d)\left[2+q_{1} g_{1}^{\prime}\left(q_{1}\right)+q_{2} g_{2}^{\prime}\left(q_{2}\right)\right] \lambda\right. & \\
& \left.+(1-2 d)\left[1+q_{1} g_{1}^{\prime}\left(q_{1}\right)\right]\left[1+q_{2} g_{2}^{\prime}\left(q_{2}\right)\right]\right\} .
\end{aligned}
$$

We know $P(1)<0$ because of $n_{2}>1$. By Jury criterion, it follows that $\left(q_{1}, q_{2}, 0,0\right)$ is unstable, and hence, system (1.4) is strongly persistent.

Similarly, we have the following result.

Theorem 4.2. Assume $s=0$ and system (1.4) is weakly persistent at time 0, then system (1.4) is weakly persistent provided the intrinsic net reproductive number $n_{2}>1$.

Remark 4.1. For system (1.2), we have the similar results to Theorems 4.1 and 4.2. From Theorems 4.1 and 4.2 we can see whether system (1.4) is strongly persistent or weakly persistent is strictly dependent on the initial data of the predator. For example, we consider the following system (4.1) for an illustration

$$
\left\{\begin{aligned}
x_{1}(t+1) & =\frac{b_{1} x_{1}(t)}{1+x_{1}(t)+\beta_{1} x_{4}(t)}+d\left[\frac{b_{2} x_{2}(t)}{1+x_{2}(t)+\beta_{2} x_{4}(t)}-\frac{b_{1} x_{1}(t)}{1+x_{1}(t)+\beta_{1} x_{4}(t)}\right] \\
x_{2}(t+1) & =\frac{b_{2} x_{2}(t)}{1+x_{2}(t)+\beta_{2} x_{4}(t)}+d\left[\frac{b_{1} x_{1}(t)}{1+x_{1}(t)+\beta_{1} x_{4}(t)}-\frac{b_{2} x_{2}(t)}{1+x_{2}(t)+\beta_{2} x_{4}(t)}\right] \\
x_{3}(t+1) & =x_{1}(t)\left[1-\frac{1}{1+\beta_{1} x_{4}(t)}\right]+x_{2}(t)\left[1-\frac{1}{1+\beta_{2} x_{4}(t)}\right] \\
x_{4}(t+1) & =b x_{3}(t)+s x_{4}(t) .
\end{aligned}\right.
$$

Choose $d=1 / 2, \beta_{1}=3, \beta_{2}=1.5, b_{1}=4, b_{2}=3, s=0, b=0.8$, it is easy to see that the intrinsic net reproductive number $n_{2}=5.4>1$. Figure 1 illustrates $x_{3}(t)+x_{4}(t)>0$ for all $t \geq 0$ with initial condition $x_{1}(0)=2, x_{2}(0)=1, x_{3}(0)=0.8, x_{4}(0)=0$, i.e., system (4.1) is only weakly persistent when initial condition is weakly persistent at time zero. Similar to Figure 1, several figures which show the system (4.1) is strongly persistent can be obtained, we omit them.

\section{Permanence of population}

We begin by presenting certain notations and preliminaries that will be used throughout this section.

(A1) $X$ is a metric space with metric $d$.

(A2) $Y$ is a closed subset of $X$.

(A3) $P: X \rightarrow X$ is continuous.

(A4) $P(Y) \subset Y,\left.P\right|_{Y}: Y \rightarrow Y$ is continuous.

(A5) $P(X \backslash Y) \subset X \backslash Y$.

Lemma 5.1. [8,11]. Suppose P satisfies $(A 1-A 5)$ and we have the following

(i) for all $x \in X, P^{t}(x)$ is a compact positive orbit;

(ii) $\left.P\right|_{Y}$ is point dissipation;

(iii) $\left.P\right|_{Y}$ has an acyclic covering $\Pi=\left\{M_{1}, M_{2}, \cdots, M_{J}\right\}$;

(iv) $W^{s}\left(M_{i}\right) \cap X \backslash Y=\emptyset$ for all $i=1,2, \cdots, J$;

then $P$ is permanent (with respect to $Y$ ). 

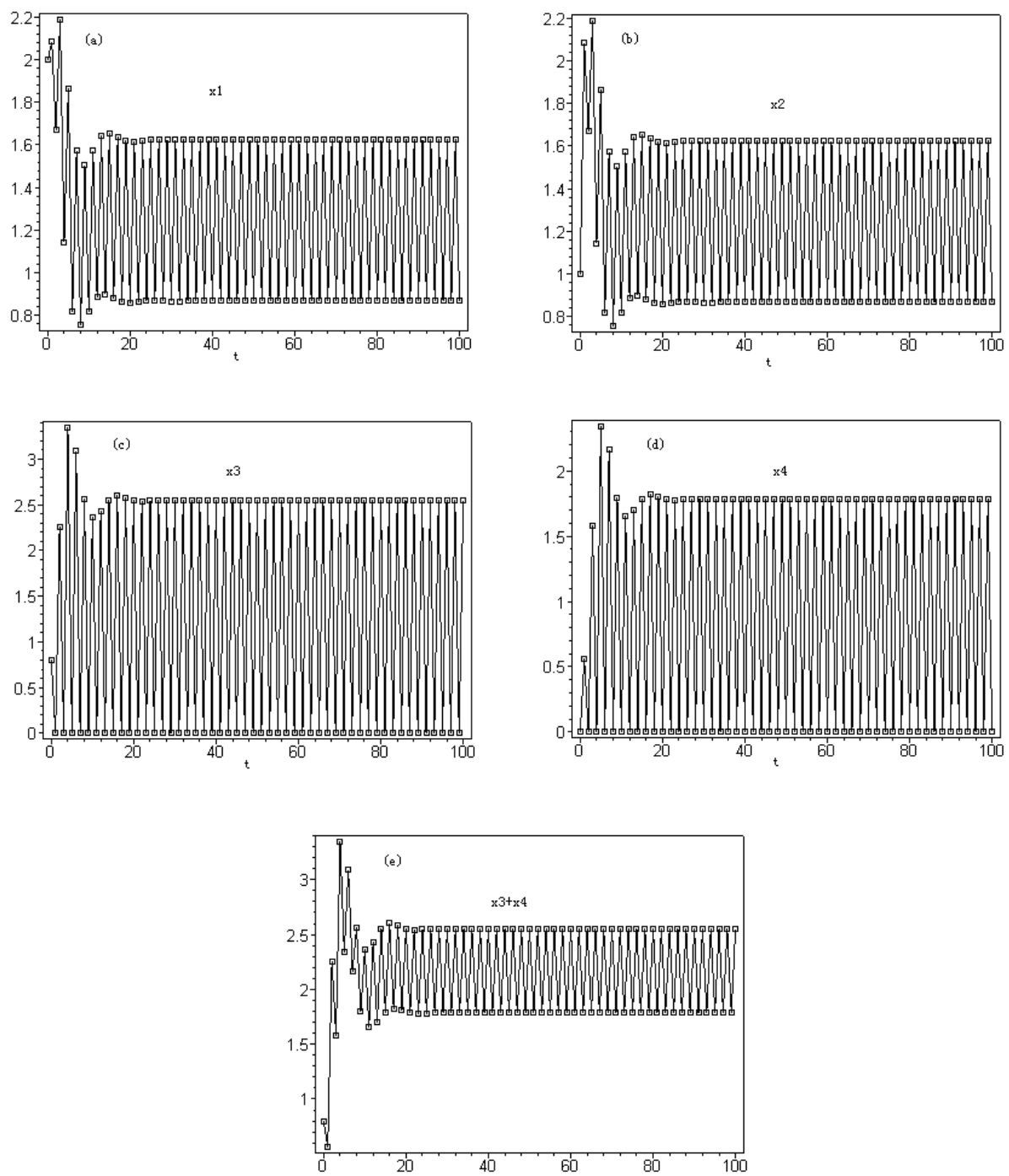

Figure $1 . d=1 / 2, \beta_{1}=3, \beta_{2}=1.5, b_{1}=4, b_{2}=3, s=0, b=0.8$ with the initial condition $x_{1}(0)=2, x_{2}(0)=1, x_{3}(0)=0.8, x_{4}(0)=0$ and $n_{2}=5.4$.

Lemma 5.2. [12]. Let $T: R_{+}^{n} \rightarrow R_{+}^{n}$ be monotone and continuous operator having the following two properties:

(a) there exists some $k$ such that for all $0<\lambda<1$ and all $x>0$

$$
\lambda T^{k} x<T^{k}(\lambda x)
$$

(b) there exists some $l$ such that $T^{l} x>0$ for all $x \geq 0(\neq 0)$ then precisely one of the following three cases does hold:

(i) each nonzero orbit is unbounded;

(ii) each orbit converges to zero, the unique fixed point of $T$;

(iii) each nonzero orbit converges to $e>0$, the unique nonzero fixed point of $T$. 
Theorem 5.1. If $0<s<1, h_{i}\left(x_{i}\right) \in S$ and system (1.4) is strongly persistent at time zero, then system (1.4) is permanent provided the intrinsic net reproductive number $n_{2}>1$ and

$$
\lambda\left[1-\frac{g_{i}\left(\beta_{i} x_{4}\right)}{g_{i}(0)}\right]<\left[1-\frac{g_{i}\left(\lambda \beta_{i} x_{4}\right)}{g_{i}(0)}\right] \quad \text { for all } 0<\lambda<1, i=1,2 \text {. }
$$

Proof. We begin by showing that the union of the $x_{1}-x_{2}$ plane and $x_{3}-x_{4}$ plane repel the positive solutions of system (1.5) uniformly. Let $X=R_{+}^{4}$, and define

$$
\begin{aligned}
& C_{1}=\left\{\left(x_{1}, x_{2}, x_{3}, x_{4}\right) \in R_{+}^{4} \mid x_{1}=x_{2}=0\right\} \\
& C_{2}=\left\{\left(x_{1}, x_{2}, x_{3}, x_{4}\right) \in R_{+}^{4} \mid x_{3}=x_{4}=0\right\}
\end{aligned}
$$

Let $Y=C_{1} \cup C_{2}$. To prove Theorem 5.1, we only need to show that all the hypotheses of Lemma 5.1 are satisfied.

From (1.4), it is easy to say that $R_{+}^{4} \backslash Y, Y$ are positive invariant under F iterations. Moreover, by Theorem 2.1, condition $(i),(i i)$ of Lemma 5.1 are clearly satisfied, thus we only need to verify the conditions (iii) and $(i v)$.

There are two constant solutions $M_{1}$ and $M_{2}$ in $Y$, corresponding respectively, to $x_{1}=x_{2}=x_{3}=x_{4}=0$ and $x_{1}=q_{1}, x_{2}=q_{2}, x_{3}=x_{4}=0$, where $\left(q_{1}, q_{2}\right)$ is a unique nonzero fixed point of system (3.2).

If $\left(u_{1}(t), u_{2}(t), u_{3}(t), u_{4}(t)\right)$ is a solution of system (1.4) initiating from $C_{1}$, then $\lim _{t \rightarrow \infty} u_{3}(t)=0, \lim _{t \rightarrow \infty} u_{4}(t)=0$. If $\left(u_{1}(t), u_{2}(t), u_{3}(t), u_{4}(t)\right)$ is a solution of system (1.4) initiating from $C_{2}$ with $u_{1}(0)>0, u_{2}(0)>0$, since $h_{i}\left(x_{i}\right) \in S$, by Theorem 3.1, we have $\lim _{t \rightarrow \infty} u_{1}(t)=q_{1}, \lim _{t \rightarrow \infty} u_{2}(t)=q_{2}$, this shows that if invariant set $M_{1}$ and invariant set $M_{2}$ are isolated, $\left\{M_{1}, M_{2}\right\}$ is isolated and is an acyclic covering.

Finally, we show (iv) holds true. We first show that $W^{s}\left(M_{1}\right) \cap R_{+}^{4} \backslash Y=\emptyset$. Suppose not, let $\left(u_{1}(t), u_{2}(t), u_{3}(t), u_{4}(t)\right)$ be a positive orbit in $R_{+}^{4} \backslash Y$ such that $\lim _{t \rightarrow \infty}\left(u_{1}(t), u_{2}(t), u_{3}(t), u_{4}(t)\right)=(0,0,0,0)$. For $i=1,2$, since $g_{i}(0)>1$, and $g_{i}\left(u_{i}+\beta_{i} u_{4}\right)>1$ for sufficiently small $u_{i}(i=1,2,3,4)$. Thus for sufficiently large $t$, we have

$$
\left\{\begin{aligned}
u_{1}(t+1) & =(1-d) u_{1} g_{1}\left(u_{1}+\beta_{1} u_{4}\right)+d u_{2} g_{2}\left(u_{2}+\beta_{2} u_{4}\right) \\
\geq & (1-d) u_{1}+d u_{2} \\
u_{2}(t+1) & =d u_{1} g_{1}\left(u_{1}+\beta_{1} u_{4}\right)+(1-d) u_{2} g_{2}\left(u_{2}+\beta_{2} u_{4}\right) \\
& \geq d u_{1}+(1-d) u_{2} .
\end{aligned}\right.
$$

Obviously, $u_{1}(t+1)+u_{2}(t+1)>u_{1}(t)+u_{2}(t)$, this contradicts with $\lim _{t \rightarrow \infty} u_{i}(t)=0(i=1,2)$, then we have $W^{s}\left(M_{1}\right) \cap R_{+}^{4} \backslash Y=\emptyset$.

Next we show that $W^{s}\left(M_{2}\right) \cap R_{+}^{4} \backslash Y=\emptyset$. Again, suppose not, let $\left(u_{1}(t), u_{2}(t), u_{3}(t), u_{4}(t)\right)$ be a positive orbit in $R_{+}^{4} \backslash Y$ such that $\lim _{t \rightarrow \infty}\left(u_{1}(t), u_{2}(t), u_{3}(t), u_{4}(t)\right)=\left(q_{1}, q_{2}, 0,0\right)$.

Since $n>1$, we can choose $\epsilon>0$ small enough such that

$$
-\frac{b}{1-s} \sum_{i=1}^{2} \frac{\beta_{i}\left(q_{i}-\epsilon\right) g_{i}^{\prime}(0)}{g_{i}(0)}>1
$$

Let $n_{0}>0$ be sufficiently large such that

$$
q_{i}-\epsilon<u_{i}(t)<q_{i}+\epsilon, \quad \text { for } \quad t \geq n_{0}, i=1,2 .
$$


Then we have, for $t \geq n_{0}$

$$
\left\{\begin{array}{l}
u_{3}(t+1) \geq\left(q_{1}-\epsilon\right)\left[1-\frac{g_{1}\left(\beta_{1} u_{4}\right)}{g_{1}(0)}\right]+\left(q_{2}-\epsilon\right)\left[1-\frac{g_{2}\left(\beta_{2} u_{4}\right)}{g_{2}(0)}\right] \\
u_{4}(t+1)=b u_{3}(t)+s u_{4}(t) .
\end{array}\right.
$$

Let us consider

$$
\left\{\begin{array}{l}
\bar{u}_{3}(t+1)=\left(q_{1}-\epsilon\right)\left[1-\frac{g_{1}\left(\beta_{1} \bar{u}_{4}\right)}{g_{1}(0)}\right]+\left(q_{2}-\epsilon\right)\left[1-\frac{g_{2}\left(\beta_{2} \bar{u}_{4}\right)}{g_{2}(0)}\right] \\
\bar{u}_{4}(t+1)=b \bar{u}_{3}(t)+s \bar{u}_{4}(t) .
\end{array}\right.
$$

We define the map $T: R_{+}^{2} \rightarrow R_{+}^{2}$

$$
\left\{\begin{aligned}
T_{1} \bar{u} & =\sum_{i=1}^{2}\left(q_{i}-\epsilon\right)\left[1-\frac{g_{i}\left(\beta_{i} \bar{u}_{4}\right)}{g_{i}(0)}\right] \\
T_{2} \bar{u} & =b \bar{u}_{3}(t)+s \bar{u}_{4}(t)
\end{aligned}\right.
$$

where $\bar{u}=\left(\bar{u}_{3}, \bar{u}_{4}\right)$. Obviously, $T$ is monotone and continuous operator, and for $l=2, k=2$, by (5.1), we have

(a) $\lambda T^{2} \bar{u}<T^{2}(\lambda \bar{u})$ for all $0<\lambda<1$ and $\bar{u}=\left(\bar{u}_{3}, \bar{u}_{4}\right)>0$;

(b) $T^{2} \bar{u}>0$ for all $\bar{u} \geq 0(\neq 0)$.

By Lemma 5.2, one of the following three cases does hold for system (5.6):

(i) each nonzero orbit is unbounded;

(ii) each orbit converges to zero, the unique fixed point of $T$;

(iii) each nonzero orbit converges to $\left(u_{3}^{*}, u_{4}^{*}\right)>0$, the unique nonzero fixed point of $T$.

In the following we show that $(i i)$ can't hold. We consider the matrix $A_{\epsilon}$ defined by

$$
A_{\epsilon}=\left(\begin{array}{cc}
0 & -\sum_{i=1}^{2} \frac{\beta_{i}\left(q_{i}-\epsilon\right) g_{i}^{\prime}(0)}{g_{i}(0)} \\
b & s
\end{array}\right)
$$

Since $A_{\epsilon}$ has an eigenvalue which is larger than 1 because of (5.3), it follows from paper [3] that the fixed point $(0,0)$ of system (5.6) is unstable. Therefore $(i i)$ can't hold. Note $A_{\epsilon}$ admits positive off-diagonal elements, the Perron-Frobenius Theorem implies that there is a positive eigenvector $v$ for the maximum eigenvalue of $A_{\epsilon}$. Let $v=\left(v_{1}, v_{2}\right)$ and let $l^{\prime}>0$ be small enough such that $l^{\prime} v_{1}<u_{3}\left(n_{0}\right), l^{\prime} v_{2}<u_{4}\left(n_{0}\right)$. If $\left(\bar{u}_{3}(t), \bar{u}_{4}(t)\right)$ is a solution of system (5.6) with $\bar{u}_{3}\left(n_{0}\right)=l^{\prime} v_{1}, \bar{u}_{4}\left(n_{0}\right)=l^{\prime} v_{2}$, then we have $u_{3}(t) \geq \bar{u}_{3}(t), u_{4}(t) \geq \bar{u}_{4}(t)$, for $t \geq n_{0}$. If $(i)$ holds, then $u_{i}(t) \rightarrow \infty\left(i=3,4\right.$, ) as $t \rightarrow \infty$, this contradicts with Theorem 2.1. If (iii) holds, let $\left(u_{3}^{*}, u_{4}^{*}\right)$ be the unique positive equilibrium of $T$, then $\lim _{t \rightarrow \infty} u_{i}(t) \geq u_{i}^{*}>0\left(i=3,4\right.$, ), this contradicts with $\lim _{t \rightarrow \infty} u_{i}(t)=0(i=3,4)$.

Consequently, system (1.4) is permanent. The proof of Theorem 5.1 is completed.

Remark 5.1. If $s=0$, then the condition (b) of Lemma 5.2 is not true. For example, we consider the point $\left(\bar{u}_{3}, 0\right)\left(\bar{u}_{3} \neq 0\right)$. Note that $T_{1}\left(\bar{u}_{3}, 0\right)=0, T_{2}\left(\bar{u}_{3}, 0\right)=b \bar{u}_{3}, T_{1}\left(0, b \bar{u}_{3}\right)=\sum_{i=1}^{2}\left(q_{i}-\epsilon\right)\left[1-\frac{g_{i}\left(\beta_{i} b \bar{u}_{3}\right)}{g_{i}(0)}\right]$ and $T_{2}\left(0, b \bar{u}_{3}\right)=0$, this gives $T^{2}\left(\partial R_{+}^{2}\right) \subset \partial R_{+}^{2}$. Therefore $T^{2}\left(\bar{u}_{3}, \bar{u}_{4}\right)>0$ is never true for $\left(\bar{u}_{3}, \bar{u}_{4}\right) \in \partial R_{+}^{2}\left(\left(\bar{u}_{3}, \bar{u}_{4}\right) \neq(0,0)\right)$. In this case, although system (1.4) isn't permanent, it must be weakly persistent (Theorem 4.2).

Corollary 5.1. Assume $0<s<1$ and system (1.2) is strongly persistent at time 0 . Then system (1.2) is permanent provided that the intrinsic net reproductive number $n_{1}>1$ and

$$
\lambda\left[1-\frac{g\left(\beta y_{2}\right)}{g(0)}\right]<\left[1-\frac{g\left(\lambda \beta y_{2}\right)}{g(0)}\right], \text { for all } 0<\lambda<1, y_{2}>0
$$


Proof. The proof of Corollary 5.1 is completely the same as that of Theorem 5.1. We only need to note that $M_{2}$ is the maximal invariant set in $\{(x, 0,0) \mid \eta \leq x \leq \hat{x}\}$.

\section{Applications AND NUMERicAl RESUlts}

In this section, we apply the general results of Section 5 to the following two-species Lotka-Volterra systems with rational growth functions or exponential growth functions:

Example 6.1. Consider the discrete model (4.1), where $g_{i}\left(x_{i}+\beta_{i} x_{4}\right)=\frac{b_{i}}{1+x_{i}+\beta_{i} x_{4}}, g_{i}\left(\beta_{i} x_{4}\right)=\frac{b_{i}}{1+\beta_{i} x_{4}}(i=1,2)$, $b_{i}, \beta_{i}, d, b, s$ are positive constants with $d=1 / 2, b_{i}>1,0 \leq b<1,0<s<1 . h_{i}\left(x_{i}\right)=\frac{b_{i} x_{i}}{1+x_{i}} \in S$ with $x_{i}^{*}=b_{i}-1>0$.

Theorem 6.1. If $n_{2}=\frac{b\left(\beta_{1}+\beta_{2}\right)\left(b_{1}+b_{2}-1\right)}{2(1-s)}>1$, then system (4.1) is permanent.

Proof. First, we show that all hypotheses of Theorem 5.1 are satisfied.

For all $0<\lambda<1, i=1,2$, since

$$
\lambda\left[1-\frac{g_{i}\left(\beta_{i} x_{4}\right)}{g_{i}(0)}\right]=\lambda\left[1-\frac{1}{1+\beta_{i} x_{4}}\right] \text { and }\left[1-\frac{g_{i}\left(\lambda \beta_{i} x_{4}\right)}{g_{i}(0)}\right]=\left[1-\frac{1}{1+\lambda \beta_{i} x_{4}}\right]
$$

then we have

$$
\left[1-\frac{1}{1+\lambda \beta_{i} x_{4}}\right]-\lambda\left[1-\frac{1}{1+\beta_{i} x_{4}}\right]=1-\lambda-\frac{1}{1+\lambda \beta_{i} x_{4}}+\frac{\lambda}{1+\beta_{i} x_{4}}
$$

Let

$$
H\left(x_{4}\right)=1-\lambda-\frac{1}{1+\lambda \beta_{i} x_{4}}+\frac{\lambda}{1+\beta_{i} x_{4}} \quad \lambda \in(0,1)
$$

since $H(0)=0$ and

$$
\begin{aligned}
H^{\prime}\left(x_{4}\right) & =\frac{-\lambda \beta_{i}}{\left(1+\beta_{i} x_{4}\right)^{2}}+\frac{\lambda \beta_{i}}{\left(1+\lambda \beta_{i} x_{4}\right)^{2}} \\
& =\lambda \beta_{i}\left[\frac{1}{\left(1+\lambda \beta_{i} x_{4}\right)^{2}}-\frac{1}{\left(1+\beta_{i} x_{4}\right)^{2}}\right] \\
& >0 \text { for } x_{4}>0
\end{aligned}
$$

then $H\left(x_{4}\right)>0$ for all $x_{4}>0, \lambda \in(0,1)$.

On the other hand, since $q_{1}=q_{2}=\frac{b_{1}+b_{2}-1}{2}>0$ and $n_{2}=\frac{b\left(\beta_{1}+\beta_{2}\right)\left(b_{1}+b_{2}-1\right)}{2(1-s)}>1$. It follows from Theorem 4.1 we have that system (4.1) is permanent.

Example 6.2. Consider the discrete model with exponential growth function

$$
\left\{\begin{aligned}
x(t+1) & =x(t) \exp \left(r(1-x(t))-\beta y_{2}(t)\right) \\
y_{1}(t+1) & =x(t)\left[1-\exp \left(-\beta r y_{2}(t)\right)\right] \\
y_{2}(t+1) & =b y_{1}(t)+s y_{2}(t)
\end{aligned}\right.
$$

where $\beta, r, b, s$ are positive constants with $0 \leq b<1,0<s<1$. For the subcommunity $x(t+1)=x(t) \exp [r(1-$ $x(t))$ ], from system (3.1) it is easy to see that there exists a constant $\eta>0$ such that $x(t) \geq \eta$ for all $t \geq 0$, and 
then we have the following Theorem:

Theorem 6.2. If $n_{1}=\frac{b \beta \eta}{1-s}>1$, then the model (6.1) is permanent.

Proof. To prove Theorem 6.1, we only need to prove (5.7) holds.

For all $0<\lambda<1$, since

$$
\lambda\left[1-\frac{g\left(\beta y_{2}(t)\right)}{g(0)}\right]=\lambda\left[1-\exp \left(-\beta y_{2}\right)\right]
$$

and

$$
\left[1-\frac{g\left(\lambda \beta y_{2}(t)\right)}{g(0)}\right]=\left[1-\exp \left(-\lambda \beta y_{2}\right)\right]
$$

then we have

$$
1-\exp \left(-\lambda \beta y_{2}\right)-\lambda\left[1-\exp \left(-\beta y_{2}\right)\right]=1-\lambda-\exp \left(-\lambda \beta y_{2}\right)+\lambda \exp \left(-\beta y_{2}\right)
$$

Let

$$
p_{1}\left(y_{2}\right)=1-\lambda-\exp \left(-\lambda \beta y_{2}\right)+\lambda \exp \left(-\beta y_{2}\right), \quad \lambda \in(0,1) .
$$

Then $p_{1}(0)=0$ and

$$
p_{1}^{\prime}\left(y_{2}\right)=\lambda \beta\left[\exp \left(-\lambda \beta y_{2}\right)-\exp \left(-\beta y_{2}\right)\right]>0 \text { for } y_{2}>0
$$

Therefore

$$
1-\exp \left(-\lambda \beta y_{2}\right)-\lambda\left[1-\exp \left(-\beta y_{2}\right)\right]>0 \text { for } y_{2}>0, \lambda \in(0,1)
$$

Thus, condition (5.7) is satisfied. From Corollary 5.1, then model (6.1) is permanent. This completes the proof.

From Theorem 6.2 we can see that if $n_{1}>1$, then system (6.1) is permanent. That is, there exists a persistent region which all orbits of system (6.1) will eventually enter and remain in it. But the dynamic behavior of solutions becomes very complex in the persistent region. As a parameter $r$ smoothly varies, the various types of behavior of the system (6.1) (such as: stable focus, two invariant curves, strange attractor) are illustrated in Figure 2.

\section{Discussion}

This paper develops a four-dimensional model for a predator-prey system where the predator has two age classes and the prey has natural barriers. The boundedness of orbits are established and then strong persistence and weak persistence are investigated. By exploiting the main theorem in $[4,8]$ and the stability trichotomy results on monotone and continuous operator in [12] we furthermore obtain the sufficient conditions for permanence of systems (1.2) and (1.4).

Models discussed by Franke et al. [24] reduce to system (1.4) when $n=4$, in [24], they mainly investigated the persistence of dominant species and extinction of species with a safe refuge. However they do not concern in persistence of system. In this paper, we establish sufficient conditions that guarantee the strong (weak) persistence, that is, if the intrinsic net reproductive number is more than one, then system (1.2-1.4) is strongly (weakly) persistent which depends on initial conditions. From a biological point of view, when adult predator gains enough food $\left(n_{1}>1\right.$ or $\left.n_{2}>1\right)$, system remains persistence. Further, if the growth functions of immature 

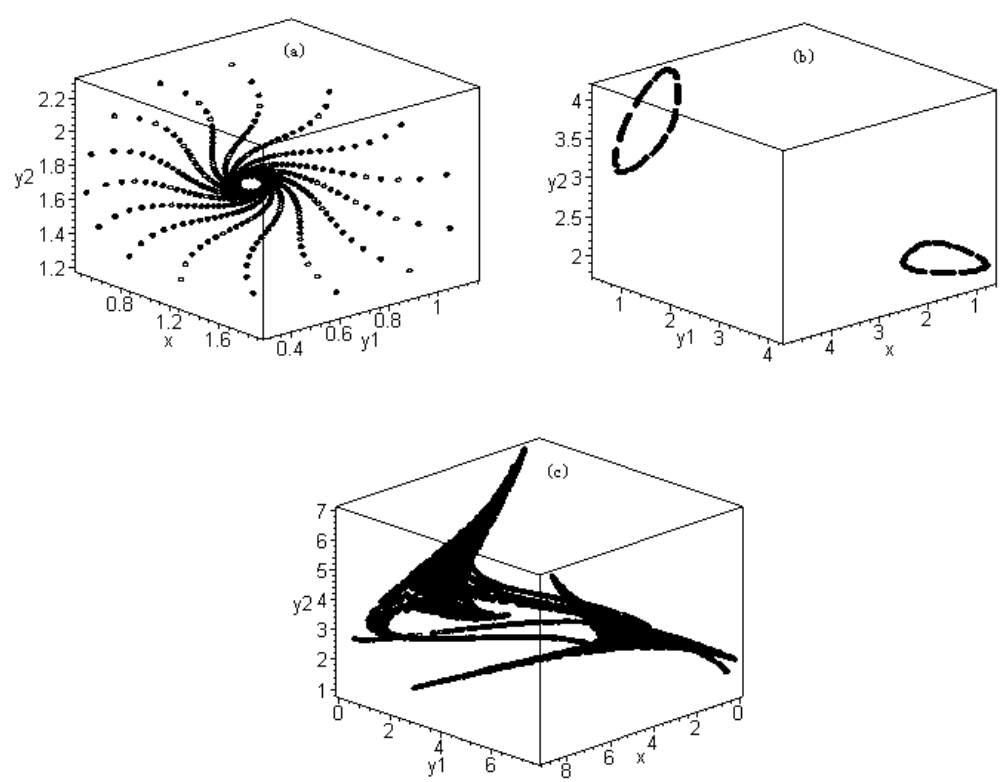

Figure 2. (a) $r=1.3, \beta=0.6, k=5, b=0.5, s=0.8$, the system (6.1) has a stable focus. (b) $r=3.25, \beta=0.6, k=5, b=0.8, s=0.4$, the system (6.1) have two invariant curves. (c) $r=3.75, \beta=0.6, k=5, b=0.8, s=0.4$, the system (6.1) has a strange attractor.
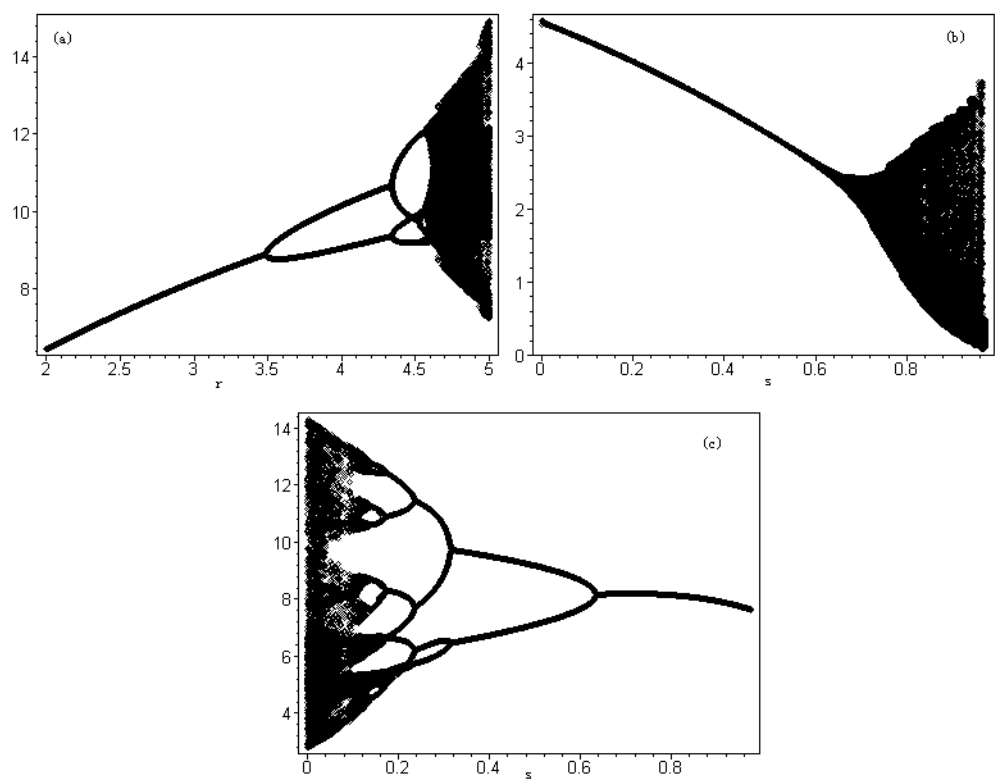

Figure 3. (a) The bifurcation diagram of the model (6.1) using parameter values with $\beta=0.4$, $k=5, b=0.4, s=0.8$. Total population size is plotted against $r$. (b) The bifurcation diagram of the model (6.1) using parameter values with $r=0.5, \beta=0.4, k=5, b=0.6$. Total population size is plotted against $s$. (c) A period-halving bifurcation diagram, plotting total population size against the adult survival rate $s$ for the model $(6.1)$ with $r=3, \beta=0.4, k=5$, $b=0.6$. 
predator is sublinear, then system (1.2-1.4) is permanent. Therefore, we develop and complete the work of [24]. Meanwhile we show that dispersal and age-structure have no effect on permanence of system. Note that survival rate of adult predator plays an important role in determining whether the system is strongly persistent or weakly persistent (see Fig. 1).

In addition, we simulate the dynamical of solutions which belong in permanent region and find that the dynamical behavior of solutions becomes very complex in the permanent region (see Fig. 2). In $[2,9,18]$, authors showed that for system (1.1) steady state is local asymptotically stable if $0<r<0.7069$, invariant curves and bifurcation occur if $0.7069<r<2.5044$ and chaos occurs if $r>2.5044$ for the given parameters $k, \beta$. Different from $[2,9,18]$, when we choose $b=0.4, s=0.8$ and fixed the other parameters which are the same as that in system (1.1), we obtain that steady state is locally asymptotically stable if $0<r<3.45$. This shows that the stable region can be expanded due to age-structure (see Fig. 3(a)). If we choose $r=0.5, b=0.6$, Figure $3(\mathrm{~b})$ shows that chaos appears suddenly from steady state as $s$ varies from 0 to 1 , which is a new feature. If we choose $r=3, b=0.6$, Figure 3(c) shows that solutions of model (6.1) move through general chaotic, period-halving bifurcation and stable equilibrium as $s$ increases. This shows that survival rate of adult predator is a sensitive parameter for discrete system with age-structure, and then indicates that age-structure makes it more complex for dynamical behavior of solutions.

From examples (6.1) and (6.2), we see that conditions (5.1) and (5.7) hold true naturally. Therefore, we conjecture that if the intrinsic net reproductive number is more than one, then system $(1.2-1.4)$ is permanent, which is more in line with reality from a biological point of view. We leave this for future works.

Acknowledgements. We would like to thank the referee and the editor for their careful reading of the original manuscript and many valuable comments and suggestions that greatly improved the presentation of this paper.

\section{REFERENCES}

[1] J.S. Allen, M.P. Moulton and F.L. Rose, Persistence in an age-structure population for a patch-type environment. Nat. Resour. Model. 4 (1990) 197-214.

[2] J.R. Beddington, C.A. Free and J.H. Lawton, Dynamical complexity in predator-prey models framed in difference equations. Nature 255 (1975) 58-60.

[3] J.F. Chen, Influence of high dimension terms for qualitative structure of solutions of a second order linear difference system with ordinary coefficient in the neighborhood of a singular point. Acta Math. Appl. Sinica (China) 11 (1988) 299-311.

[4] M.E. Clark and L.J. Gross, Periodic solutions to nonautonomous difference equations. Math. Biosci. 102 (1990) $105-119$.

[5] J.M. Cushing, An introduction to structured population dynamics. SIAM Soc. Indus. Appl. Math., Philadelphia (1998).

[6] H.I. Freedman and J.W.-H. So, Persistence in discrete models of a population which may be subjected to harvesting. Nat. Resour. Model. 2 (1987) 135-145.

[7] H.I. Freedman and Y. Takeuchi, Global stability and predator dynamics in a model of prey dispersal in a patchy environment. Nonlinear Anal. TMA 13 (1989) 993-1002.

[8] H.I. Freedmen and W.H. Josephso, Persistence in discrete semidynamical systems. SIAM J. Math. Anal. 20 (1989) 930-938.

[9] I. Gumowski and C.Mira, Recurrences and discrete dynamics systems. Lect. Notes Math. 809 (1980) 61-96.

[10] A. Hastings, Complex interactions between dispersal and dynamics: Lessons from coupled logistic equations. Ecology 74 (1993) $1362-1372$

[11] V. Hutson and K. Schmitt, Permanence and the dynamics of biological systems. Math. Biosci. 111 (1992) 1-71.

[12] U. Krause and P. Ranet, A limit set trichotomy for monotone nonlinear dynamical systems. Nonlinear Anal. TMA 19 (1992) 375-392.

[13] Y. Kuang and Y. Takeuchi, Predator-prey dynamics in models of prey dispersal in two patch environments. Math. Biosci. 120 (1994) 77-98.

[14] N.R. Leblond, Porcupine caribou herd. Canadian Arctic Resources Comn., Offuwa (1979).

[15] S.A. Levin, Dispersion and population interactions. Amer. Natur. 108 (1974) 207-228.

[16] J. Li, Persistence in discrete age-structure population models. Bull. Math. Biol. 50 (1992) 351-366.

[17] A. Okubo, Diffusion and ecological problems, math. models. Springer, Berlin (1980).

[18] K. Schumacher, Regions and oscillations in second order predator-prey recurrences. J. Math. Biol. 16 (1983) $221-231$.

[19] J.F. Selgrade and G. Namkoong, Stable periodic behavior in a pioneer-climax model. Nat. Resour. Model. 4 (1990) $215-227$. 
[20] J.G. Skellam, Random dispersal in theoretical populations. Biometrika 38 (1951) 196-218.

[21] H.L. Smith, Cooperative systems of differential equations with concave nonlinearities. Nonlinear Anal. TMA 10 (1986) 10371052.

[22] Y. Takeuchi, Cooperative systems theory and global stability of diffusion models. Acta Appl. Math. 14 (1989) $49-57$.

[23] W.D. Wang and L.S. Chen, A predator-prey system with stage-structure for predator. Comput. Math. Appl. 33 (1997) 83-91.

[24] A.-A. Yakubu, Prey dominance in discrete predator-prey system with a prey refuge. Math. Biosci. 144 (1997) $155-178$.

To access this journal online: www.edpsciences.org 